\title{
Decreased expression of Annexin A10 in gastric cancer and its overexpression in tumor cell growth suppression
}

\author{
JEONG KYU KIM $^{1 *}$, PUM JOON KIM ${ }^{2 *}$, KWANG HWA JUNG ${ }^{1}$, JI HEON NOH ${ }^{1}$, \\ JUNG WOO EUN ${ }^{1}$, HYUN JIN BAE ${ }^{1}$, HONG JIAN XIE ${ }^{1}$, JIN MEI SHAN ${ }^{3}$, \\ WANG YING PING ${ }^{3}$, WON SANG PARK ${ }^{1}$, JUNG YOUNG LEE ${ }^{1}$ and SUK WOO NAM ${ }^{1}$ \\ ${ }^{1}$ Department of Pathology, Microdissection Genomics Research Center; ${ }^{2}$ Department \\ of Cardiology, College of Medicine, The Catholic University of Korea, Seoul, Korea; \\ ${ }^{3}$ Department of Pathology, First Hospital Jilin University, Changchun, P.R. China
}

Received March 26, 2010; Accepted June 2, 2010

DOI: $10.3892 /$ or_00000898

\begin{abstract}
Gastric carcinoma is the most common neoplasm in Southeast Asian populations and is the second leading cause of cancer death worldwide. Annexins are a family of cytosolic calcium and membrane binding proteins that have been implicated in a wide variety of cell functions. Recent studies have suggested that Annexin A10 (ANXA10), a member of the Annexin protein family, is down-regulated in specific types of cancer. However, the underlying molecular mechanisms of the dysregulation of ANXA10 remain to be elucidated. In the present study, to investigate the biological effects of ANXA10 on gastric carcinoma, aberrant expression of ANXA10 was evaluated by Western blot analysis, reverse transcriptase-polymerase chain reaction (RT-PCR) and immunohistochemistry (IHC), in gastric cancer tissues and cell lines. Decreased expression of ANXA10 was observed in five selected gastric cancer tissues compared to the normal surrounding mucosa. In the cancer cell lines, seven out of nine selected gastric cancer cell lines had no detectable ANXA10 by RT-PCR. Among these, when an ANXA10 expressing plasmid was introduced into MKN-1 cells, cell growth was suppressed and apoptosis augmented. The results of this study demonstrated that ANXA10 was aberrantly regulated in gastric carcinoma and suggests that down-regulation of ANXA10 might be involved in gastric carcinogenesis. In addition, ANXA10 may play a role, as a tumor suppressor, in the development and progression of gastric cancer.
\end{abstract}

Correspondence to: Dr Suk Woo Nam, Department of Pathology, College of Medicine, The Catholic University of Korea, \#505 Banpodong, Seocho-gu, Seoul 137-701, Korea

E-mail: swnam@catholic.ac.kr

${ }^{*}$ Contributed equally

Key words: Annexin A10, calcium-binding protein, gastric carcinoma, apoptosis, cell growth

\section{Introduction}

Gastric cancer is the most common malignancy in Korea and some of the other Southeast Asian populations; it is the second leading cause of cancer death worldwide (1). However, the mechanism associated with the development of gastric cancer and progression has not been determined. Carcinogenesis is a multi-step process that is associated with alterations of cellular oncogenes and tumor suppressor genes necessary for malignant transformation (2-4). In gastric carcinogenesis, it has also been suggested that multiple genetic alterations are responsible for the development and progression of gastric cancer, and alterations in specific genes that play an important role in diverse cell activity such as cell adhesion, signal transduction, differentiation, development and DNA-repair; genes for such functions have been identified (5-8). However, many of these putative oncogenes or tumor suppressor genes require further study as future targets for therapeutic interventions in patients with gastric cancer. Recent studies have confirmed chromosomal alterations in gastric cancer. The loss of many chromosomal loci such as: $1 \mathrm{p}, 2 \mathrm{p}, 3 \mathrm{p}, 4 \mathrm{p}, 5 \mathrm{q}$, $6 \mathrm{q}, 7 \mathrm{q}, 8 \mathrm{q}, 12 \mathrm{q}, 13 \mathrm{p}, 14 \mathrm{q}, 17 \mathrm{p}, 17 \mathrm{q}$ and $18 \mathrm{q}$ have been found in gastric cancers (9-13). However, the mechanisms of loss, of these loci and certain genes, are poorly understood. Furthermore, it is unclear how these genetic changes correspond to the clinical characteristics of individual patients with gastric cancer. Therefore, improvements in diagnostic, protective and treatment strategies require a better understanding of the various mechanisms underlying gastric carcinogenesis.

Annexins (ANXs) are a family of cytosolic calciumregulated proteins and membrane-binding proteins that have been implicated in diverse biological processes that link ANX to many membrane-related events, such as the regulated organization of membrane domains and/or membranecytoskeleton links, certain exocytic and endocytic transport steps and the regulation of ion fluxes across membranes (14). The activity of these proteins are mediated by $\mathrm{Ca}^{2+}$ dependent signal transduction pathways also involved in the regulation of the cell cycle, growth, vesicle trafficking, and apoptosis (14-19). Moreover, recent studies have shown that ANXs are 
implicated in complex mechanisms, and abnormal expression of ANX protein levels have been correlated with tumor progression. In various cancers, certain ANXs are thought to participate in carcinogenesis. Expression of ANXA1, 2, 7, 8 and 11 has been reported to be up- or down-regulated, ANXA3, 4 and 6 to be up-regulated and ANXA10 to be down-regulated in specific types of cancer (20). The downregulation of the ANXA10 protein, was first identified in higher vertebrates, and has been correlated with the presence of a p53 mutation and poor prognosis in patients with hepatocellular carcinoma (21). Recently, ANXA10 expression was found to be reduced in mucinous adenocarcinoma, and low ANXA10 expression was also found to be associated with a poor prognosis (13). These results suggest that ANXA10, at least in part, participates in the process of tumor progression.

In the present study, to investigate the biological role of ANXA10 in gastric carcinogenesis, expression of ANXA10 in gastric cancer was compared to corresponding normal stomach tissues and was also confirmed in gastric carcinoma cell lines. In addition, to explore the biological role of ANXA10 in gastric cancer, a recombinant ANXA10 was constructed and in vitro tumor growth and apoptosis assays performed.

\section{Materials and methods}

Tissue samples and tissue microarray. Five frozen human gastric cancers and their corresponding normal tissues were obtained from Jilin University; the samples were randomly selected for this study. Approval was obtained from the institutional review board of the Jilin University of China. Informed consent was provided according to the Declaration of Helsinki. For the tissue microarray (TMA), the intestinaltype was present in 40 cases, 62 were the diffuse-type and 20 were the mixed-type of gastric cancer. The mean tumor size was $6.37 \mathrm{~cm}$ and 122 tumors were located in the middle or lower section of the stomach. For the TMA construction, a total of 122 gastric cancer specimens from formalin fixed, paraffin-embedded gastric cancer samples were obtained from the archives of the Department of Pathology at our institution. Two pathologists screened the histological sections and selected areas representative of the tumor cells. Two and one tissue core samples, from each cancer and normal tissue, were taken and placed in a new recipient paraffin block using a commercially available microarray instrument (Beecher Instruments, Micro-Array Technologies, Silver Spring, MD, USA), according to established methods (22). Approval was obtained from the institutional review board of the Catholic University of Korea, College of Medicine. There was no evidence of familial cancer in any of the patients.

Cell culture and reagents. MKN-1, MKN-28, MKN-74, SNU-216, SNU-484, SNU-638, SNU-668 and SNU-719 cells were purchased from the Korean Cell Line Bank (KCLB; Seoul, Korea) and AGS cells were purchased from the American Type Culture Collection (ATCC; Manassas, VA, USA). Each cell line was grown in RPMI-1640 medium supplemented with $10 \%$ fetal bovine serum (Sigma, St. Louis, MO, USA). MKN-1 cells were trypsinized, and then seeded at $2 \times 10^{5}$ cells/plate in a $60-\mathrm{mm}$ dish, and allowed to grow overnight at $37^{\circ} \mathrm{C}$ in a humidified incubator with $5 \% \mathrm{CO}_{2}$. At $24 \mathrm{~h}$ after plating, the cells were transfected with ANXA10specific siRNAs in Opti-MEM (Life Technologies, Gaithersburg, MD, USA). Transfection was carried out using oligofectamine reagent (Invitrogen, Carlsbad, CA, USA) according to the manufacturer's specifications.

Reverse transcriptase-polymerase chain reaction ( $R T-P C R)$. The total RNA from the MKN-1 cells was isolated using TRIzol reagent (Invitrogen) according to the manufacturer's instructions. The RNA concentration was determined by NanoDrop (Thermo Fisher Scientific Inc., Waltham, MA, USA). Next, $1 \mu \mathrm{g}$ of total RNA was reverse transcribed using the Superscript II enzyme (Gibco-BRL, Gaithersburg, MD, USA) with $2.5 \mu \mathrm{M}$ oligo (d)T (Amersham Biosciences, Piscataway, NJ, USA). The reaction mixture was incubated at $42^{\circ} \mathrm{C}$ for $60 \mathrm{~min}$ and then at $72^{\circ} \mathrm{C}$ for $15 \mathrm{~min}$. To normalize differences in the amount of total cDNA added to each reaction, glyceraldehydes-3-phosphate dehydrogenase (GAPDH) gene expression was used as an endogenous control. The cycling conditions were: initial denaturation at $95^{\circ} \mathrm{C}$ for $10 \mathrm{~min}$, followed by 40 cycles at $95^{\circ} \mathrm{C}$ for $15 \mathrm{sec}, 58^{\circ} \mathrm{C}$ for $5 \mathrm{sec}, 72^{\circ} \mathrm{C}$ for $5 \mathrm{sec}$. ANXA10 and GAPDH transcripts were measured using agarose gel electrophoresis.

Western blot analysis. The following antibodies were used: anti-ANXA10, -GAPDH, - $\alpha$-tubulin (Santa Cruz Biotechnology, Santa Cruz, CA, USA), anti-PARP (Cell Signaling Technology, Danvers, MA, USA), anti-mouse IgG (Amersham Biosciences), anti-rabbit IgG (Bio-Rad Laboratories, Hercules, CA, USA) and the ECL plus Western blotting detection system (Amersham Biosciences) were used to detect the bound antibodies. The intensity of the Western blot bands was quantified using the LAS 3000 (Fuji Photo Film Co., Tokyo, Japan).

Full-length human Annexin A10 (ANXA10) construct. The full-length cDNA encoding ANXA10 of the SNU-620 cells was amplified by PCR with the ANXA10 primer: 5'-TGG TGGAATTCATGTTTTGTGGAGACTATGAGCA-3' (forward); 5'-GGCCGCTCGAGTTAGTAGTCCTCAGC ATCACCAG-3' (reverse) EcoRI and XhoI restriction sites introduced into the primers. The resulting PCR products were digested with EcoRI and XhoI, purified, and inserted into the pcDNA 3.1/HisC vector. The recombinant plasmids (pcDNA 3.1/HisC_ANXA10-1,-3) were confirmed by sequencing.

Cell counts for cell growth. The effect on cell growth was determined by counting cells at the indicated time with the use of a hemocytometer. Briefly, the MKN-1 cells were seeded in 12-well flat-bottomed microtiter plates at a density of $2 \times 10^{4}$ cells per well. The cells were transfected with recombinant plasmid containing ANXA10 or a Mock vector, and then the cells were allowed to grow for the indicated time. The cells were trypsinized and then were counted using staining with trypan blue $(0.8 \mathrm{mM}$ in PBS). All measurements were performed in triplicate and each experiment was repeated at least 3 times.

Apoptosis analysis. The Annexin V-FITC Apoptosis Detection kit I (BD Biosciences, San Jose, CA, USA) was used to 
A

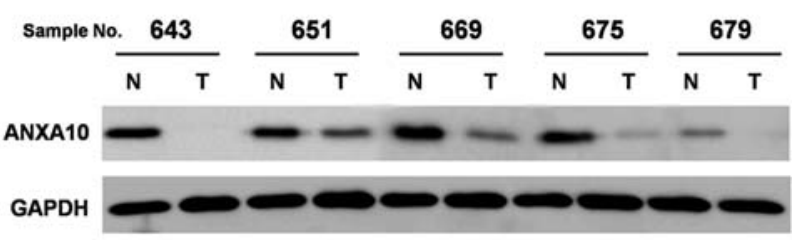

B

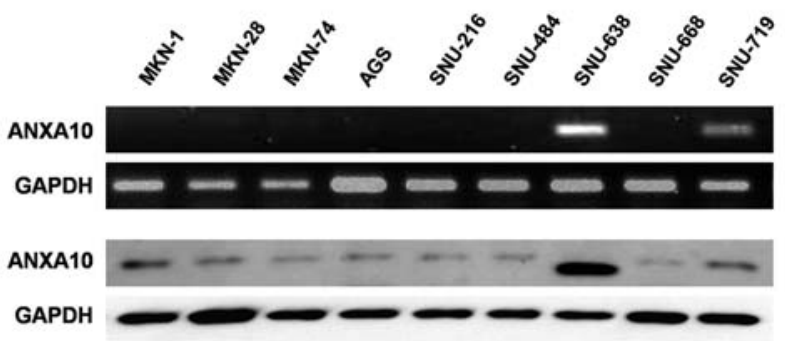

Figure 1. Expression of ANXA10 in gastric carcinoma tissues and cell lines: Human gastric tissues from five patients with gastric cancer (patients are shown by number and identified by one letter: $\mathrm{N}$, corresponding normal tissue; T, tumor tissue), nine of the gastric cancer cell lines were collected and harvested for protein or RNA. (A) Western blot analysis showing the expression of ANXA10 protein in gastric cancer tissues. GAPDH served as an internal control (bottom). Data are representative of at least two independent experiments. (B) RT-PCR and Western blot analysis confirmed the expression of ANXA10 mRNA and protein in gastric cancer cell lines, respectively. Data are representative of at least two independent experiments

quantify the level of apoptosis in the samples. Briefly, after transfection, the cells were trypsinized, washed twice with cold PBS and resuspended in $1 \mathrm{X}$ binding buffer at a concentration of $1 \times 10^{6}$ cells $/ \mathrm{ml}$, and $100 \mu 1$ of the solution $\left(1 \times 10^{5}\right.$ cells $)$ was transferred to a $5 \mathrm{ml}$ culture tube and $5 \mu \mathrm{l}$ of Annexin V-FITC solution was added; $10 \mu \mathrm{l}$ of PI was added to each tube and the cells were analyzed by flow cytometry.
Statistical analysis. Each experiment was performed at least three times. The data are presented as the mean \pm standard errors for the number of experiments. Statistical significance was defined by the results of the unpaired Student's t-test at a $\mathrm{P}<0.05$ and the Chi-square test for immunohistochemistry.

\section{Results}

Decreased expression of ANXA10 in gastric cancers. ANXA10 is located on chromosome 4 at the q33 band (26); chromosome $4 \mathrm{q}$ is one of the most common regions with a high frequency of allelic loss in HCC. Recent studies have shown that reduced expression of ANXA10 is associated with gastric carcinoma. However, its role in gastric carcinoma has not been clearly defined. Thus, in order to investigate the aberrant expression of ANXA10 in gastric carcinoma, the expression levels of ANXA10 in both gastric cancer cell lines and human gastric cancer tissues were measured and compared. In the gastric cancer cell lines, when the expression of ANXA10 by RT-PCR and Western blot analysis was assessed, most of the cancer cell lines derived from gastric carcinoma showed a reduced expression of ANXA10.

In the gastric cancer cells, ANXA10 was not detectable by both RT-PCR and Western blot analysis; with the exception of the SNU-620 and SNU-719 cell lines (Fig. 1B). In order to confirm the aberrant expression of ANXA10 in gastric carcinoma, the differential expression of ANXA10 was determined in five selected human gastric carcinoma tissues. As shown in Fig. 1A, expression of the ANXA10 protein level was found to be reduced in the tumor tissues compared to the corresponding normal tissues. The protein expression of ANXA10 was further validated by immunostaining of the gastric cancer specimens with ANXA10 antibodies; the results are summarized in Fig. 2 and Table I. Briefly, 31 out of the 122 gastric carcinomas tested $(25.4 \%)$ had negative or weak positive staining with the ANXA10 antibodies, while 102 out of the $122(83.6 \%)$ normal gastric tissues showed moderate or strong expression of ANXA10 in the normal adjacent tissues. Fig. 2 shows a representative image of normal and gastric carcinoma tissues (Fig. 2A), magnified

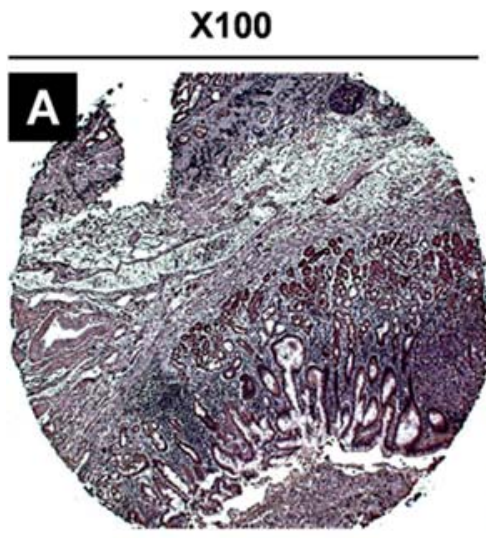

Block 1-27

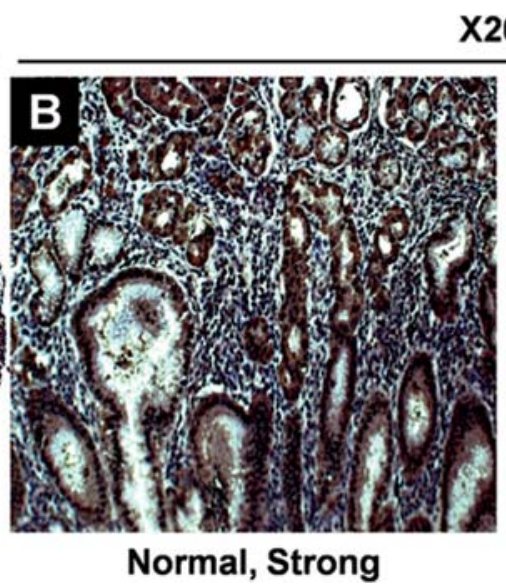

X200

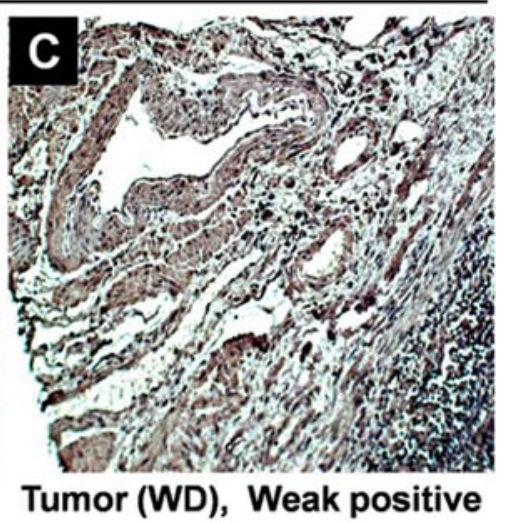

Figure 2. Representative image of immunohistochemistry for ANXA10 expression in human gastric cancer and adjacent normal tissues: Immunostaining of gastric cancer and adjacent normal tissues was carried with ANXA10 antibodies (1:50 dilution). (A) A representative image of gastric cancer and adjacent normal tissues with magnification x100. (B) adjacent normal tissue with magnification x200, and (C) gastric cancer with magnification x200 was developed with DAB chromogen and counterstained with hematoxylin. 
A

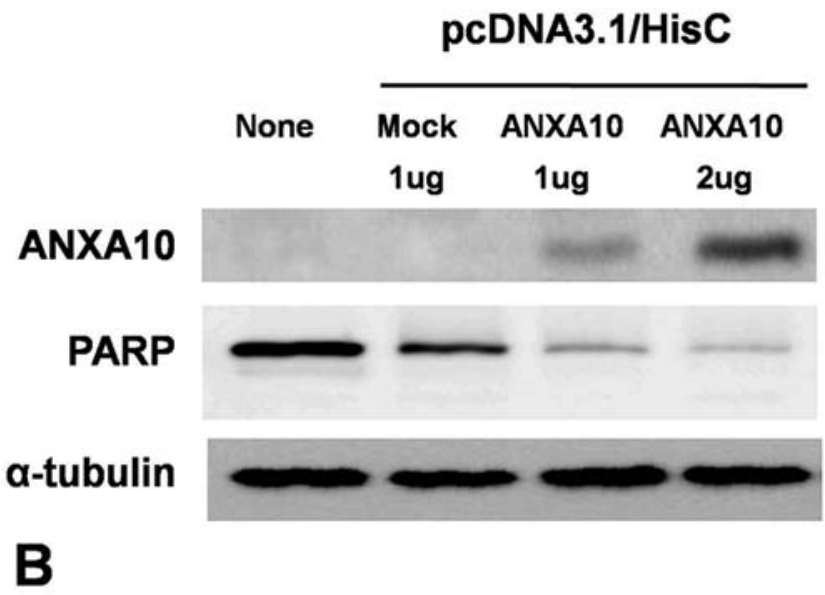

\section{Cell counting}

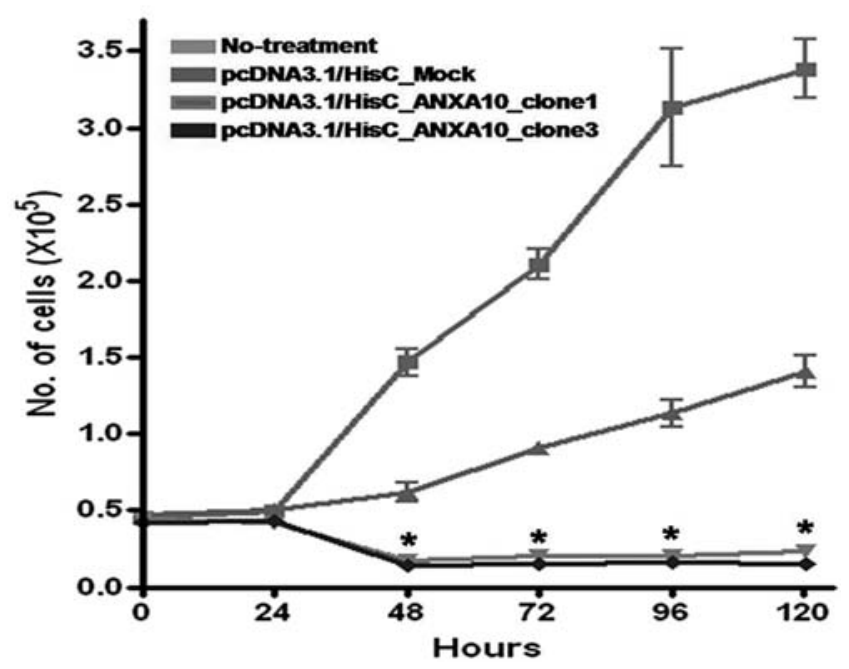

Figure 3. Introduction of ANXA10 expression plasmid into MKN-1 cells inhibits their proliferation. (A) Western blot analysis showing the overexpression of ANXA10 protein levels in MKN-1 cells after transfection with the indicated concentration of recombinant plasmid expressing ANXA10, but not with the control plasmid (Mock). The cleavage form of PARP, a hallmark of apoptosis, was assessed by Western blotting with antibodies to PARP. (B) Cell growth rates of MKN-1 cells transiently transfected with pcDNA3.1/HisC_Mock, pcDNA3.1/HisC_ANXA10clone 1 and -clone3. The cells were seeded on 24-well plates at an equal density and cultured for the indicated time before the number of cells was evaluated by cell counting. The data are presented as the mean \pm standard error for the three experiments (unpaired Student t-test, ${ }^{*} \mathrm{p}<0.05$ vs. control).

normal gastric tissues (Fig. 2B) and gastric cancer tissues (Fig. 2C).

Overexpression of ANXA10 inhibits cell growth and induces apoptosis of $M K N-1$ cells. To determine the biological consequences of dysregulation of ANXA10 expression, whether ANXA10 was a suppressor of growth in a gastric cancer cell line was evaluated. MKN-1 cells, which did not express ANXA10, were transiently transfected with a control plasmid (pcDNA3.1/HisC) or with an ANXA10 plasmid (pcDNA3.1/ HisC_ANXA10-clone1, -clone3). As shown in Fig. 3A, introducing an ANXA10 expression vector into the MKN-1 ells was associated with effective expression of ANXA10 in
Table I. Result of immunohistochemical staining of ANAA10 in gastric cancer.

\begin{tabular}{lcc}
\hline P-value & Normal $(\mathrm{n}=122)$ & $\begin{array}{c}\text { Tumor }(\mathrm{n}=122) \\
\mathrm{P}=0.0029^{\mathrm{a}}\end{array}$ \\
\hline $\begin{array}{l}\text { ANXA10, } \mathrm{n}(\%) \\
\begin{array}{l}\text { ANXA10 negative } \\
\text { (-, weak positive) }\end{array}\end{array}$ & $20(16.4 \%)$ & $31(25.4 \%)$ \\
$\begin{array}{l}\text { ANXA10 positive } \\
\text { (moderate) }\end{array}$ & $69(56.6 \%)$ & $78(64 \%)$ \\
$\begin{array}{l}\text { ANXA10 negative } \\
\text { (strong) }\end{array}$ & $33(27 \%)$ & $13(10.6 \%)$ \\
\hline
\end{tabular}

${ }^{\mathrm{a} C h i-s q u a r e}$ test.

the MKN-1 cells compared to the non-expressing (Mock) vector. To determine the biological effects of overexpression of ANXA10, the growth rate of the MKN-1 cells was determined using the cell counting assay. As shown in Fig. 3B, overexpression of ANXA10 significantly reduced cell growth compared to cells treated with the pcDNA3.1/HisC_Mock vector. The anti-mitogenic effects on the MKN-1 cells could be partially explained by the augmentation of cellular senescence or apoptosis. Indeed, cleavage of poly-ADP-ribose polymerase (PARP) was observed when ANXA 10 was transfected, a hallmark of apoptosis (Fig. 3A).

To confirm the apoptotic potential of AXA10, cells that were transfected with ANXA10 were stained with Annexin V/ propidium iodide (PI), and compared to the control by flow cytometric analysis. As shown in Fig. 4, the flow cytometry analysis with PI and Annexin V staining, for the dead cells or apoptotic cells, indicated that both early and late stages of apoptosis (M2) were enhanced by introducing ANXA10 into the MKN-1 cells. A significant induction of apoptotic cells was observed in both clones with the ANXA10 expression vector compared to the cells treated with the pcDNA3.1/ HisC_Mock vector (none or Mock vector treatment). The apoptotic cells (right in dot-plot graphs of Fig. 4) increased from $40.67 \%$ to $50.69 \%$ in the pcDNA3.1/HisC_ANXA10clone 1 and pcDNA3.1/HisC_ANXA10-clone3, ANXA10 expression vector groups, compared to no treatment (17.97\%) or Mock vector $(17.56 \%)$ treatment.

\section{Discussion}

The ANXs are a multigene family of closely related calcium binding proteins without EF-hands (the helix-loop-helix calcium-binding motif) and charged phospholipids of membrane binding proteins that show type specific expression (23). There are at least 12 human ANX members, designated A1, A2, A3, A4, A5, A6, A7, A8, A9, A10, A11, and A13 (20). The ANXs shares a similar structure that is characterized by the presence of four or eight repeats of a 70-amino acid motif and a highly variable amino terminal end (23-25). Among the more than 13 ANX family members, different ANXs have unique tissue and cell distribution in vertebrates. ANXA2, -A5, 

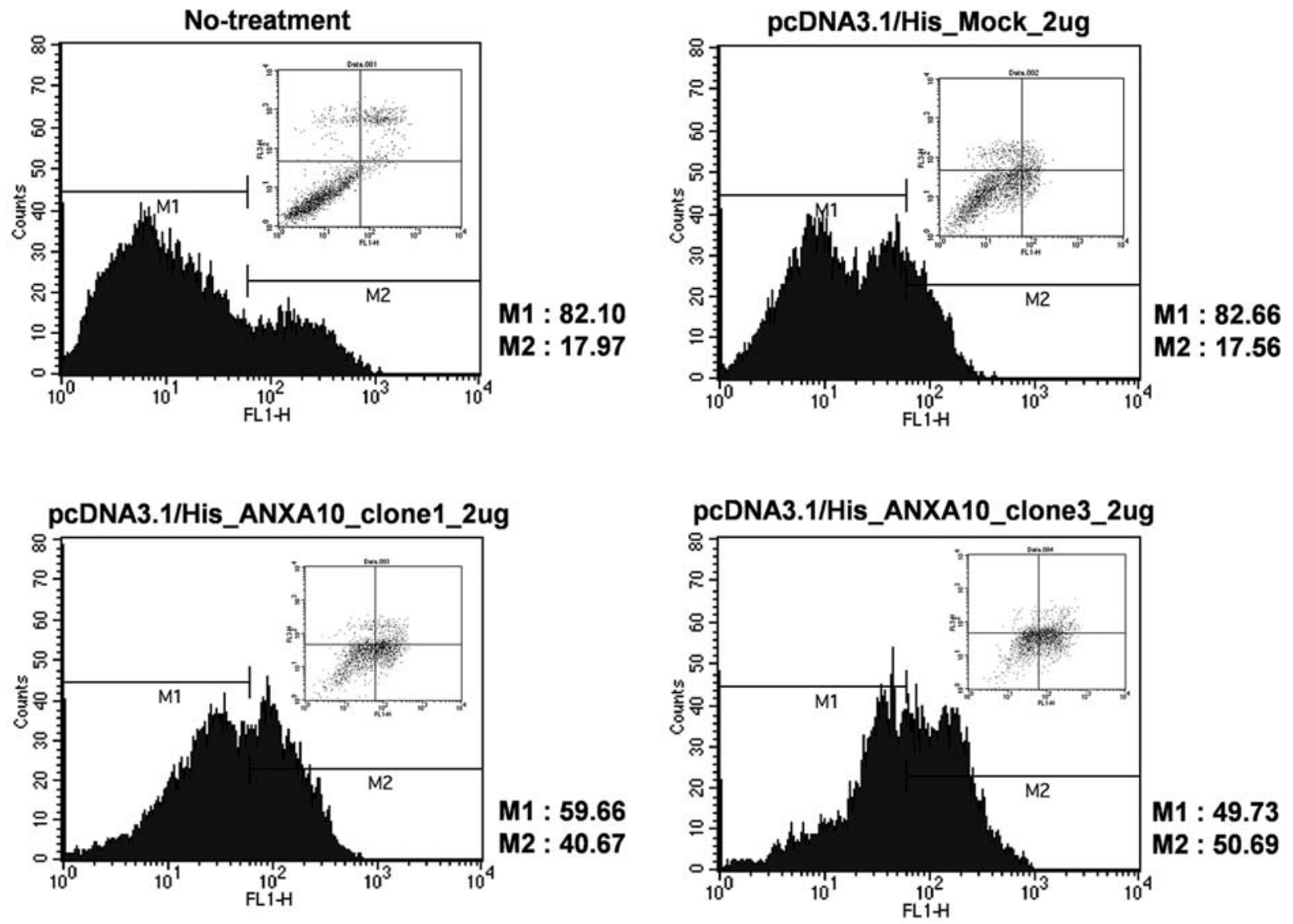

Figure 4. Increase in the portion of apoptotic MKN-1 cells overexpressing ANXA10: The MKN-1 cells were transiently transfected with pcDNA3.1/HisC_Mock or pcDNA3.1/HisC_ANXA10-clone1 or -clone3 and stained with Annexin V and propidium iodide and then quantified by fluorescence-activated cell sorter (FACS) analysis. The population shown in the image is the total distribution of cells. Significant induction of the apoptosis portion (M2) was observed in the MKN-1 cells overexpressing ANXA10 in both clones. Data were obtained from three independent experiments.

-A6, and -A11 are ubiquitous, whereas ANXA3,-A8, and -A13 have a more restricted tissue distribution $(23,26,27)$. Some ANXs are overexpressed in several types of cancers, while others show loss of expression.

There is also increasing evidence to indicate that the changes in ANX expression and/or subcellular localization of ANXs contribute to the development and progression of cancer. Several of the ANX genes are located in chromosomal regions that show a high frequency of loss in specific types of cancer, suggesting that these ANXs may be tumor suppressor genes $(20,28,29)$. ANXA10, a member of the ANX family, has several distinct features, including rare expression, codon deletion in conserved repeat 3 , and an unusual ablation of the type II calcium binding sites in tetrad core repeats (30).

ANXA10 has been recently considered a potential gene therapy target for hepatocellular carcinoma due to its reduced expression associated with vascular invasion, early recurrence, and poor prognosis associated with the p53 mutation (21); it is located on chromosome 4 at band q33 (31). Chromosome $4 \mathrm{q}$ is one of the most common regions associated with a high frequency of allelic loss in hepatocellular carcinoma (32-35). However, despite the accumulating evidence supporting aberrant expression of ANXA10 and a high frequency of allelic loss of the ANXA10 gene, in specific types of cancer $(21,36)$, there is no information on its biological activity in various types of cancer, especially in gastric carcinoma.
ANXA10 expression has been shown to be reduced in gastric carcinoma based on the findings of previous studies. In addition, large-scale molecular changes in colon cancer have been suggested by expression profiling (37). In the present study, the results showed down-regulation of ANXA10 in gastric carcinomas; in addition, recombinant plasmid-mediated protein overexpression of ANXA10 suppressed the growth properties of MKN-1 cancer cells. The analysis of endogenous expression of ANXA10, in the gastric cancer cell lines, revealed no detectable mRNA, with the exception of SNU-638 and -719 (Fig. 1B).

With regard to the biological activity of ANXA10 during tumor progression, there are several lines of evidence that indicate that ANXA10 acts as an anti-proliferative and antiprosurvival factor in several types of cancer cells including hepatocellular carcinomas and gastric cancer cells $(13,21)$. The results of this study showed dysregulation of ANXA10 in gastric carcinoma. To determine the biological role of ANXA10 in gastric carcinoma, recombinant plasmid-mediated gene overexpression was employed. The overexpression of endogenous ANXA10, in the MKN-1 cells, resulted in the regression of cell growth (Fig. 3B) and increased the fraction of apoptotic cells (Fig. 4). According to a recent study, silencing of endogenous ANXA10 stimulated the migration rate of cells and increased the number of colonies of the MKN-1 cells, which was then decreased when transfected with expression plasmid cDNA (13). The results of this study 
are consistent with previous findings that suggest that ANXA10 might act as a tumor suppressor in gastric cancer cells by restraining cell growth and inducing basal apoptosis.

To estimate the clinical significance of ANXA10 expression, gastric carcinoma specimens were analyzed using an immunostaining method; the results showed that expression of the ANXA10 protein was slightly decreased in 31 out of the 122 gastric carcinomas tested $(25.4 \%)$, and resulted in negative or weak positive staining with the ANXA10 antibody. However, 20 out of the $122(16.4 \%)$ normal gastric tissues showed negative or weak positive staining (Fig. 2 and Table I). ANXA10 expression was found to be reduced in mucinous adenocarcinoma suggesting that the loss of ANXA10 may reflect the loss of exocytotic function during the dedifferentiation process in gastric carcinogenesis. Low ANXA10 expression was associated with a poor prognosis in patients with HCC and gastric carcinoma $(13,21)$.

In conclusion, the results of this study showed reducedexpression of ANXA10 in both cancer cell lines and human gastric carcinomas. In addition, overexpression of ANXA10 in gastric cancer cell lines suppressed tumor cell growth and induced apoptosis. These results suggest that loss of ANXA10 expression may contribute to tumor cell growth and survival. To confirm these results, additional target proteins and their biological activity must be studied.

\section{Acknowledgements}

This study was supported by Basic Science Research Program through the National Research Foundation of Korea (NRF) funded by the Ministry of Education, Science and Technology (2009-0072504) and by a grant of the Korea Healthcare technology R\&D Project, Ministry for Health, Welfare and Family Affairs, Republic of Korea (A084930) and by the Korean Science and Engineering Foundation via the Cell Death Disease Research Center at The Catholic University of Korea.

\section{References}

1. Parkin DM, Pisani P and Ferlay J: Estimates of the worldwide incidence of 25 major cancers in 1990. Int J Cancer 80: 827-841, 1999.

2. Parkin DM, Pisani P and Ferlay J: Global cancer statistics. CA Cancer J Clin 49: 33-64, 1999.

3. Fearon ER and Vogelstein B: A genetic model for colorectal tumorigenesis. Cell 61: 759-767, 1990.

4. Kinzler KW and Vogelstein B: Life (and death) in a malignant tumour. Nature 379: 19-20, 1996.

5. Park WS, Lee JH, Shin MS, et al: Inactivating mutations of the caspase-10 gene in gastric cancer. Oncogene 21: 2919-2925, 2002.

6. Park WS, Oh RR, Park JY, et al: Somatic mutations of the trefoil factor family 1 gene in gastric cancer. Gastroenterology 119 : 691-698, 2000.

7. Werner M, Becker KF, Keller G and Hofler H: Gastric adenocarcinoma: Pathomorphology and molecular pathology. J Cancer Res Clin Oncol 127: 207-216, 2001.

8. Fuchs CS and Mayer RJ: Gastric carcinoma. N Engl J Med 333: $32-41,1995$.

9. Sano T, Tsujino T, Yoshida K, et al: Frequent loss of heterozygosity on chromosomes 1q, 5q, and $17 \mathrm{p}$ in human gastric carcinomas. Cancer Res 51: 2926-2931, 1991.

10. Uchino S, Tsuda H, Noguchi M, et al: Frequent loss of heterozygosity at the DCC locus in gastric cancer. Cancer Res 52: 3099-3102, 1992.
11. Semba S, Yokozaki H, Yasui W and Tahara E: Frequent microsatellite instability and loss of heterozygosity in the region including BRCA1 (17q21) in young patients with gastric cancer. Int J Oncol 12: 1245-1251, 1998.

12. Wang Y, Zheng E and Ke Y: Studies of loss of heterozygosity $(\mathrm{LOH})$ in chinese human gastric cancer tissues. Zhonghua Zhong Liu Za Zhi 20: 116-118, 1998.

13. Kim J, Kim MA, Jee CD, Jung EJ and Kim WH: Reduced expression and homozygous deletion of annexin A10 in gastric carcinoma. Int J Cancer 125: 1842-1850, 2009.

14. Gerke V, Creutz CE and Moss SE: Annexins: Linking $\mathrm{Ca}^{2+}$ signalling to membrane dynamics. Nat Rev Mol Cell Biol 6: 449-461, 2005 .

15. Hayes MJ and Moss SE: Annexins and disease. Biochem Biophys Res Commun 322: 1166-1170, 2004.

16. Gerke V and Moss SE: Annexins: from structure to function. Physiol Rev 82: 331-371, 2002.

17. Hayes MJ, Longbottom RE, Evans MA and Moss SE: Annexinopathies. Subcell Biochem 45: 1-28, 2007.

18. Rand JH: The annexinopathies: A new category of diseases. Biochim Biophys Acta 1498: 169-173, 2000.

19. Rescher U and Gerke V: Annexins - unique membrane binding proteins with diverse functions. J Cell Sci 117: 2631-2639, 2004.

20. Mussunoor S and Murray GI: The role of annexins in tumour development and progression. J Pathol 216: 131-140, 2008.

21. Liu SH, Lin CY, Peng SY, et al: Down-regulation of annexin A10 in hepatocellular carcinoma is associated with vascular invasion, early recurrence, and poor prognosis in synergy with p53 mutation. Am J Pathol 160: 1831-1837, 2002.

22. Kononen J, Bubendorf L, Kallioniemi A, et al: Tissue microarrays for high-throughput molecular profiling of tumor specimens. Nat Med 4: 844-847, 1998.

23. Smith PD and Moss SE: Structural evolution of the annexin supergene family. Trends Genet 10: 241-246, 1994.

24. Dubois T, Oudinet JP, Mira JP and Russo-Marie F: Annexins and protein kinases C. Biochim Biophys Acta 1313: 290-294, 1996.

25. Benz J and Hofmann A: Annexins: from structure to function. Biol Chem 378: 177-183, 1997.

26. Raynal P and Pollard HB: Annexins: The problem of assessing the biological role for a gene family of multifunctional calciumand phospholipid-binding proteins. Biochim Biophys Acta 1197: 63-93, 1994

27. Morgan RO and Fernandez MP: Annexin gene structures and molecular evolutionary genetics. Cell Mol Life Sci 53: 508-515, 1997.

28. Srivastava M, Bubendorf L, Srikantan V, et al: ANX7, a candidate tumor suppressor gene for prostate cancer. Proc Natl Acad Sci USA 98: 4575-4580, 2001.

29. Garcia Pedrero JM, Fernandez MP, Morgan RO, et al: Annexin A1 down-regulation in head and neck cancer is associated with epithelial differentiation status. Am J Pathol 164: 73-79, 2004.

30. Morgan RO, Jenkins NA, Gilbert DJ, et al: Novel human and mouse annexin A10 are linked to the genome duplications during early chordate evolution. Genomics 60: 40-49, 1999.

31. Deloukas P, Schuler GD, Gyapay G, et al: A physical map of 30,000 human genes. Science 282: 744-746, 1998.

32. Yeh SH, Chen PJ, Lai MY and Chen DS: Allelic loss on chromosomes $4 \mathrm{q}$ and $16 \mathrm{q}$ in hepatocellular carcinoma: Association with elevated alpha-fetoprotein production. Gastroenterology 110: 184-192, 1996.

33. Nagai H, Pineau P, Tiollais P, Buendia MA and Dejean A: Comprehensive allelotyping of human hepatocellular carcinoma. Oncogene 14: 2927-2933, 1997.

34. Bando K, Nagai H, Matsumoto S, et al: Identification of a 1-cM region of common deletion on $4 \mathrm{q} 35$ associated with progression of hepatocellular carcinoma. Genes Chromosomes Cancer 25: 284-289, 1999.

35. Wong N, Lai P, Lee SW, et al: Assessment of genetic changes in hepatocellular carcinoma by comparative genomic hybridization analysis: Relationship to disease stage, tumor size, and cirrhosis. Am J Pathol 154: 37-43, 1999.

36. Matsubara D, Niki T, Ishikawa S, et al: Differential expression of S100A2 and S100A4 in lung adenocarcinomas: Clinicopathological significance, relationship to p53 and identification of their target genes. Cancer Sci 96: 844-857, 2005.

37. Koh KH, Rhee H, Kang HJ, et al: Differential gene expression profiles of metastases in paired primary and metastatic colorectal carcinomas. Oncology 75: 92-101, 2008. 